\title{
Interaction of Magnetic Field and Nonlinear Convection in the Stagnation Point Flow over a Shrinking Sheet
}

\author{
Rakesh Kumar and Shilpa Sood \\ Department of Mathematics, Central University of Himachal Pradesh, TAB, Shahpur, Kangra, Himachal Pradesh 176206, India \\ Correspondence should be addressed to Rakesh Kumar; rakesh.lect@gmail.com
}

Received 27 November 2015; Revised 26 March 2016; Accepted 28 March 2016

Academic Editor: Yuanxin Zhou

Copyright ( 2016 R. Kumar and S. Sood. This is an open access article distributed under the Creative Commons Attribution License, which permits unrestricted use, distribution, and reproduction in any medium, provided the original work is properly cited.

The steady two-dimensional boundary layer stagnation point flow due to a shrinking sheet is analyzed. The combined effects of magnetic field and nonlinear convection are taken into account. The governing equations for the flow are modeled and then simplified using the similarity transformation and boundary layer approach. The numerical solution of the reduced equations is obtained by the second-order finite difference scheme also known as Keller box method. The influence of the pertinent parameters of the problem on velocity and temperature profiles, skin friction, and sheet temperature gradient are presented through the graphs and tables and discussed. The magnetic field and nonlinear convection parameters significantly enhance the solution range.

\section{Introduction}

The analysis of the hydromagnetic flow over stretching or shrinking surfaces is very demanding due to wide range of its applications in industry, physics, and engineering sciences including bioengineering. The impact of magnetic field on the flow of an electrically conducting viscous fluid finds its applications in purification of crude oil, glass manufacturing, paper production, polymer sheets, MHD electrical power generation, magnetic material processing, and so forth [1]. Moreover, the final product relies on the rate of cooling, which is decided by the configuration of the boundary layer near the stretching/shrinking sheet. Chakrabarti and Gupta [2] investigated the hydromagnetic flow and heat transfer over a stretching surface. Zhang and Wang [3] presented a rigorous mathematical analysis to analyze the MHD flow of power law fluid over a stretching sheet. The axially symmetric stagnation point flow of an electrically conducting fluid under transverse magnetic field was examined by Kakutani [4]. An analysis for three-dimensional stagnation point flow over a stretching surface was made by Attia [5] considering magnetic field and heat generation. Ali et al. [6] extended the above paper by considering the induced magnetic field. Recently, Ali et al. [7] have reported on the effects of mixed convection parameter and magnetic field over a vertical stretching sheet in the neighborhood of the stagnation point. Mahapatra et al. [8] investigated the MHD stagnation point flow of power law fluid over a sheet which is stretching in its own plane with a velocity proportional to the distance from the stagnation point. Very recently, Khan et al. [9] analyzed the thermodiffusion effects on the MHD stagnation point flow of nanofluid over a stretching sheet. Hayat et al. [10] investigated the stagnation point flow on a non-Newtonian fluid over a stretching sheet. Some significant aspects of the MHD stagnation point flow over stretching surfaces can be found in Hayat et al. [11], Shateyi and Makinde [12], Ibrahim et al. [13], Mahapatra and Gupta [14], Ishak et al. [15], and so forth. In recent times, the researchers are attracted towards the flow over shrinking surfaces. These flows are different from the flow over stretching surfaces in many ways. In shrinking sheet problems, the surface of the sheet is stretched towards a slot and hence generating a velocity away from the sheet. Therefore, the generated vorticity does not remain within the boundary layer and the flow will be unlikely to exist [16]. Wang [17] confirmed that the solutions can be found only for small shrinking rates and multiple solutions may exist for two-dimensional cases. Mahapatra and Nandy [18] were the researchers who analyzed that if suitable suction or stagnation point is added, then the vorticity can be controlled and the similarity solution will exist. Moreover, the 
stagnation region is the region which faces the highest pressure, heat transfer, and rates of mass deposition [19]. Keeping this in mind, the buoyancy effects on the MHD stagnation point flow of nanofluid were discussed by Makinde et al. [20]. Zaimi et al. [21] examined the boundary layer flow and heat transfer for nanofluid over a nonlinearly stretching/shrinking sheet. Akbar et al. [22] obtained the dual numerical solutions for the MHD stagnation point flow of Carreau fluid towards a permeable shrinking sheet. Some contributions on the study of stagnation point flow over shrinking surfaces for various physical situations and different fluids appeared in literature. Some of them can be found in Yian et al. [23], Bachok et al. [24], Nadeem et al. [25], Bhattacharyya et al. [26], Van Gorder et al. [27], Mansur et al. [28], and Ishak et al. [29]. However, for viscous fluid flows with heat transfer, the influence of linear dependence of density on temperature, that is, free convection, is found to be highly substantial in applications relating to industrial manufacturing processes and, therefore, cannot be ignored. But when the temperature difference between the surface and the ambient fluid is substantially large, the nonlinear density temperature (NDT) variations in the buoyancy force term have a significant effect on the flow and heat transfer features. Vajravelu and Sastri [30] discussed the flow between two parallel plates by considering the quadratic density temperature (QDT) variation and showed that the flow and heat transfer rates are substantially affected by it. Bhargava and Agarwal [31] examined the fully developed free convection flow in circular pipe with nonlinear density temperature variations. The nonlinear convection effects on the flow past a flat porous plate have been reported by Vajravelu et al. [32]. The flow dynamics of shrinking surfaces is still unknown and many of its characteristics are yet to be investigated. Motivated by this, the aim is to target the interaction between magnetic field and nonlinear convection on the stagnation point flow over a shrinking sheet.

\section{Formulation of the Problem}

Consider a steady two-dimensional mixed convection flow of an incompressible, electrically conducting, and viscous fluid over a shrinking sheet as shown in Figure 1. Here, we have considered the Cartesian coordinate system with fixed origin such that the $x$-axis is along the direction of the shrinking surface and the $y$-axis is taken normal to the sheet. A magnetic field of uniform strength $B_{0}$ is applied normal to shrinking sheet and magnetic Reynolds number is assumed to be small to neglect the induced magnetic field. Here, we have supposed that external fluid velocity is $U_{e}(x)=a x$, where $a>0$ is the stagnation flow strength, and velocity of the sheet is $U_{w}(x)=c x$, where $c<0$ represents shrinking of the sheet and $c>0$ represents stretching of the sheet. We have also assumed that fluid has an ambient temperature $T_{\infty}$ and $T=T_{w}(x)$ as temperature of the sheet.

The governing boundary layer equations under the abovementioned assumptions are as follows.

Equation of continuity that is

$$
\frac{\partial u}{\partial x}+\frac{\partial v}{\partial y}=0
$$

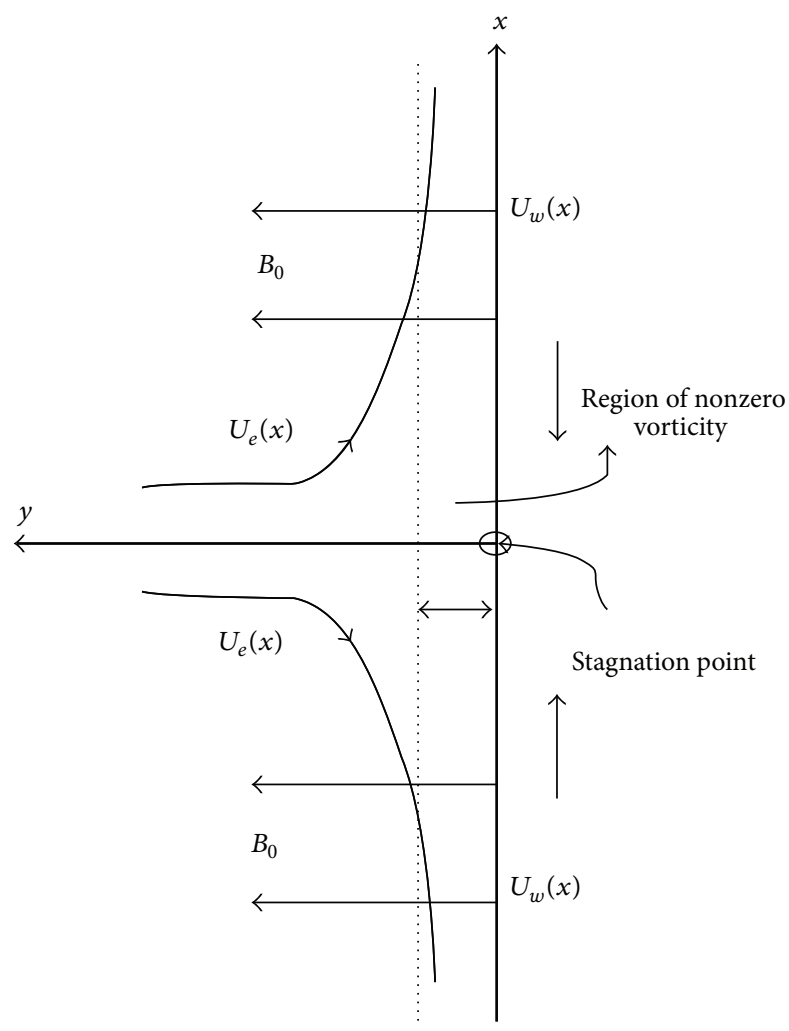

Figure 1: Schematic diagram of the problem.

Equation of motion that is

$$
\begin{aligned}
u \frac{\partial u}{\partial x}+v \frac{\partial u}{\partial y}= & U_{e} \frac{\partial U_{e}}{\partial x}+v \frac{\partial^{2} u}{\partial y^{2}}+\frac{\sigma B_{0}^{2}}{\rho}\left(U_{e}-u\right) \\
& +g \beta\left(T-T_{\infty}\right)+g \beta_{1}\left(T-T_{\infty}\right)^{2}
\end{aligned}
$$

Equation of energy that is

$$
u \frac{\partial T}{\partial x}+v \frac{\partial T}{\partial y}=\alpha \frac{\partial^{2} T}{\partial y^{2}}
$$

where $u(x, y)$ is the velocity component along the $x$-direction and $v(x, y)$ is the velocity component along the $y$-direction, $\nu$ is the kinematic viscosity of the fluid, $T$ is the fluid temperature, $\alpha$ is thermal diffusivity, $\beta$ and $\beta_{1}$ are the coefficients of thermal expansion, $g$ is acceleration due to gravity, and $B_{0}$ is the applied magnetic field.

Suitable boundary conditions for this particular flow problem are

$$
\begin{aligned}
& v=0, \\
& u=U_{w}(x)=c x, \\
& T=T_{w}(x)=T_{\infty}+b x,
\end{aligned}
$$

$$
\text { at } y=0
$$




$$
\begin{aligned}
& u=U_{e}(x)=a x, \\
& T=T_{\infty},
\end{aligned}
$$$$
\text { as } y \longrightarrow \infty \text {. }
$$

In order to reduce above set of nonlinear partial differential equations (1)-(3) with given boundary conditions into a set of ordinary differential equations, the following similarity transformations will be used:

$$
\begin{aligned}
\eta & =\sqrt{\frac{U_{e}}{v x}} y, \\
\psi & =\sqrt{a v} x f(\eta), \\
\theta(\eta) & =\frac{T-T_{\infty}}{T_{w}-T_{\infty}}, \\
u & =\frac{\partial \psi}{\partial y} \\
v & =-\frac{\partial \psi}{\partial x} .
\end{aligned}
$$

Here $\psi$ is the stream function, which identically satisfies the continuity equation, and $\theta$ is the dimensionless temperature.

The transformed set of nonlinear ordinary differential equations is

$$
\begin{aligned}
& f^{\prime \prime \prime}(\eta)+f(\eta) f^{\prime \prime}(\eta)-\left[f^{\prime}(\eta)\right]^{2}+1 \\
& \quad+M\left[1-f^{\prime}(\eta)\right]+\lambda \theta(\eta)[1+\gamma \theta(\eta)]=0, \\
& \theta^{\prime \prime}(\eta)+\operatorname{Pr}\left[f(\eta) \theta^{\prime}(\eta)-\theta(\eta) f^{\prime}(\eta)\right]=0,
\end{aligned}
$$

where prime denotes differentiation with respect to $\eta$. The nondimensional parameters announced in (6) are $M=$ $\sigma B_{0}^{2} / \rho a$ (magnetic parameter), $\operatorname{Pr}=\nu / \alpha$ (Prandtl number), and $\lambda=\mathrm{Gr}_{x} / \operatorname{Re}_{x}^{2}$ (buoyancy parameter), where $\mathrm{Gr}_{x}=$ $g \beta\left(T_{w}-T_{\infty}\right) x^{3} / \nu^{2}$ (local Grashof number), $\mathrm{Re}_{x}=x U_{e} / \nu$ (local Reynolds number), and $\gamma=\beta_{1}\left(T_{w}-T_{\infty}\right) / \beta$ (NDT parameter).

With the use of the abovementioned similarity transformations, boundary conditions (4) reduce to

$$
\begin{aligned}
& f(\eta)=0, \\
& f^{\prime}(\eta)=\frac{c}{a}=s, \quad \theta(\eta)=1, \\
& \text { at } \eta=0, \\
& f^{\prime}(\eta)=1, \\
& \theta(\eta)=0,
\end{aligned}
$$

where $c / a$ is the velocity ratio parameter (ratio of shrinking velocity rate to the straining velocity rate).
In this problem the physical parameters of interest are skin friction $C_{f}$ and local Nusselt number $\mathrm{Nu}_{x}$ which are defined as follows:

$$
\begin{aligned}
C_{f} & =\frac{\tau_{w}}{\rho U_{e}^{2}}, \\
\mathrm{Nu}_{x} & =\frac{x q_{w}}{k\left(T_{w}-T_{\infty}\right)},
\end{aligned}
$$

where $\tau_{w}=\mu(\partial u / \partial y)_{y=0}$ and $q_{w}=-k(\partial T / \partial y)_{y=0}$ are shear stress and heat flux at the sheet, respectively. Here $\mu$ is the dynamic viscosity and $k$ is the thermal conductivity. By using nondimensional similarity transformations (5), we get

$$
\begin{aligned}
C_{f} \operatorname{Re}_{x}^{1 / 2} & =f^{\prime \prime}(0), \\
\mathrm{Nu}_{x} \mathrm{Re}_{x}^{-1 / 2} & =-\theta^{\prime}(0) .
\end{aligned}
$$

\section{Solution Methodology}

The above set of coupled nonlinear ordinary differential equations (6) along with the boundary conditions (7) are solved numerically by means of an implicit finite difference scheme known as Keller box method combined with Newton's linearization method [32-35]. This method has a second-order accuracy and is unconditionally stable. The method has the following steps to solve a system of nonlinear ordinary differential equations:

(1) Reduce the given equations into the first order.

(2) Write finite difference approximation of the ordinary differential equations using central difference derivatives.

(3) Linearize the nonlinear system of equations using Newton's linearization method and write it in matrixvector form.

(4) Use LU decomposition technique to solve the block tridiagonal structure.

\section{Results and Discussion}

In the present analysis, rigorous computations have been performed in order to achieve two targets, namely, understanding the influence of pertinent parameters on flow and heat transfer characteristics and deriving the numerical range of velocity ratio parameters to ensure the laminar flow over the considered shrinking sheet. The numerical results have been obtained using the symbolic language in MATLAB. To retain the accuracy in numerical calculations, a step size of $\Delta \eta=0.01$ was found to be suitable to obtain accuracy within tolerance error less than $10^{-5}$ in all cases. The position of edge of the boundary layer $\eta_{\infty}$ and step size $\Delta \eta$ in $\eta$ has to be adjusted for different values of parameters to retain the accuracy. To check accuracy of the present results, a comparison has been made with the data existing in literature and is presented in Table 1, which confirms the accuracy of this numerical method. Inspired by the accuracy of results, the present study is carried out to comprehend the 
TABLE 1: Comparison of the values of skin friction $f^{\prime \prime}(0)$ for $\gamma=0$, $\lambda=0$, and $M=0$.

\begin{tabular}{|c|c|c|c|c|}
\hline$s$ & Solutions & Present result & Mahapatra et al. [36] & Wang [17] \\
\hline \multirow{2}{*}{-0.25} & Upper & 1.402253 & 1.402242 & 1.40224 \\
\hline & Lower & - & - & - \\
\hline \multirow{2}{*}{-0.50} & Upper & 1.495685 & 1.495672 & 1.49567 \\
\hline & Lower & - & - & - \\
\hline \multirow{2}{*}{-0.75} & Upper & 1.489316 & 1.489296 & 1.48932 \\
\hline & Lower & - & - & - \\
\hline \multirow{2}{*}{-1.00} & Upper & 1.328840 & 1.328819 & 1.32882 \\
\hline & Lower & 0.0 & 0.0 & \\
\hline \multirow{2}{*}{-1.10} & Upper & 1.186707 & 1.186680 & - \\
\hline & Lower & 0.049309 & 0.049222 & - \\
\hline \multirow{2}{*}{-1.15} & Upper & 1.082262 & 1.082232 & 1.08223 \\
\hline & Lower & 0.117298 & 0.116702 & 0.116702 \\
\hline \multirow{2}{*}{-1.20} & Upper & 0.932512 & 0.932470 & - \\
\hline & Lower & 0.233638 & -0.233648 & - \\
\hline \multirow{2}{*}{-1.24} & Upper & 0.584900 & 0.584374 & - \\
\hline & Lower & 0.553728 & 0.554215 & - \\
\hline \multirow{2}{*}{0.0} & Upper & 1.2325975 & 1.233588 & 1.232588 \\
\hline & Lower & - & - & - \\
\hline \multirow{2}{*}{0.1} & Upper & 1.1465699 & 1.146560 & 1.146560 \\
\hline & Lower & - & - & - \\
\hline \multirow{2}{*}{0.2} & Upper & 1.0511379 & 1.051131 & 1.051130 \\
\hline & Lower & - & - & - \\
\hline \multirow{2}{*}{2.00} & Upper & -1.887316 & -1.887308 & -1.88731 \\
\hline & Lower & - & - & - \\
\hline \multirow{2}{*}{5.00} & Upper & -10.26479 & -10.26475 & -10.26475 \\
\hline & Lower & - & - & - \\
\hline
\end{tabular}

impacts of physical parameters entering into this study, like magnetic field, buoyancy force, nonlinear convection, and Prandtl number, on the stagnation point flow over a shrinking sheet. Arbitrary values are allotted to the related physical parameters.

Here, we are pointing towards a study on the stagnation point flow over a shrinking sheet which was investigated by Wang [17]. In the present analysis, it is worth mentioning that the solution range extends significantly with the introduction of NDT parameter $(\gamma)$ and its interaction with the magnetic field; and hence the solutions are also found to be existing for larger shrinking rates. Moreover, Miklavcic and Wang [37] showed that multiple solutions exist for the flow over a shrinking sheet and in our analysis we have also encountered dual solution regimes. The dual solutions range obtained in terms of velocity ratio parameters $\left(s_{1}, s_{2}\right)$ for different values of nonlinear convection parameter $(\gamma)$ and magnetic field $(M)$ has been depicted through Table 2. This table clearly communicates that $\left(s_{1}, s_{2}\right)$ extends significantly for skin friction $f^{\prime \prime}(0)$ and rate of heat transfer $-\theta^{\prime}(0)$ with the increasing $\gamma$. However, the increasing strength of magnetic field $(M)$ exhibits stable expansion at the left end and significant contraction at the right end of $\left(s_{1}, s_{2}\right)$. The streamlines have been plotted in Figure 2 for negative value of velocity

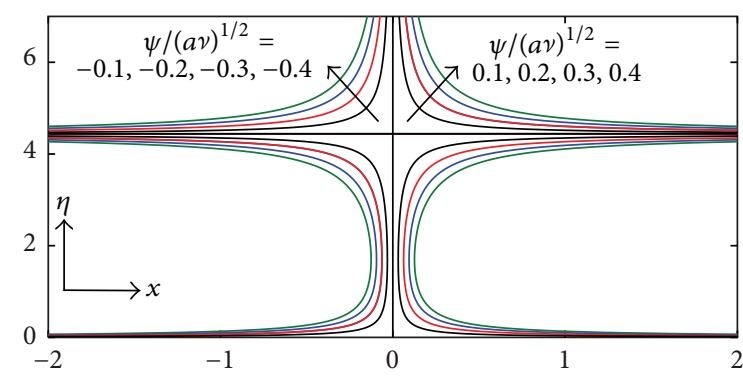

FIGURE 2: Variation of streamlines for $s=-3$, Pr $=3, M=0.2$, $\gamma=0.5$, and $\lambda=0.5$.

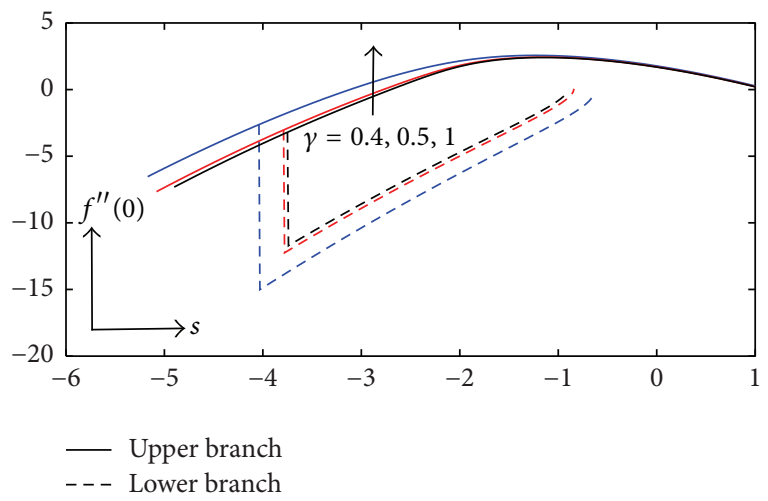

FIGURE 3: Variation of skin friction with $s$ for various values of $\gamma$ with $M=0.5, \lambda=0.5$, and $\operatorname{Pr}=1$.

TABLE 2: Range of dual solutions for skin friction and rate of heat transfer with $\lambda=0.5$ and $\operatorname{Pr}=1$.

\begin{tabular}{lccc}
\hline$s$ & $\gamma=0.4$ & $\gamma=0.5$ & $\gamma=1$ \\
\hline$f^{\prime \prime}(0) /\left(s_{1}, s_{2}\right)$ & $(-3.73,-0.90)$ & $(-3.75,-0.84)$ & $(-4.04,-0.64)$ \\
$-\theta^{\prime}(0) /\left(s_{1}, s_{2}\right)$ & $(-3.73,-0.90)$ & $(-3.75,-0.84)$ & $(-4.04,-0.64)$ \\
\hline & $M=0.0$ & $M=0.2$ & $M=0.5$ \\
\hline$f^{\prime \prime}(0) /\left(s_{1}, s_{2}\right)$ & $(-3.72,-0.51)$ & $(-3.75,-0.65)$ & $(-3.79,-0.84)$ \\
$-\theta^{\prime}(0) /\left(s_{1}, s_{2}\right)$ & $(-3.72,-0.51)$ & $(-3.75,-0.65)$ & $(-3.79,-0.84)$ \\
\hline
\end{tabular}

ratio parameter $(s)$, and it is observed that a horizontal line divides the streamlines into two regions and a reverse flow pattern is obtained on the shrinking surface.

The variations of skin friction $f^{\prime \prime}(0)$ and rate of heat transfer $-\theta^{\prime}(0)$ with nonlinear convection parameter $(\gamma)$ and magnetic field $(M)$ are illustrated in Figures 3-6. The dual nature of solutions has been depicted by these figures for negative values of velocity ratio parameters, that is, shrinking of the sheet. The small neighborhoods around the left end points of $\left(s_{1}, s_{2}\right)$ obtained in Table 2 are the bifurcation points. These bifurcation points turn out to be the singular points for the second solution profiles of $f^{\prime \prime}(0)$ and $-\theta^{\prime}(0)$. This is due to the physical fact that the nonlinear convection parameter generates severe velocity and temperature gradients. It is also found from these figures that both $f^{\prime \prime}(0)$ and $-\theta^{\prime}(0)$ are enhanced for the first solution with the increasing $\gamma$ and $M$. However for the second solution, $f^{\prime \prime}(0)$ and $-\theta^{\prime}(0)$ are reduced with the increasing $\gamma$. The second solution profiles of 


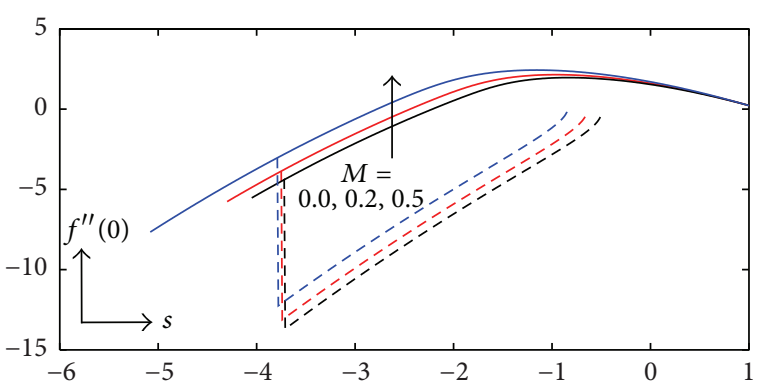

- Upper branch
--- Lower branch

FIGURE 4: Variation of skin friction with $s$ for various values of $M$ with $\gamma=0.5, \lambda=0.5$, and $\operatorname{Pr}=1$.

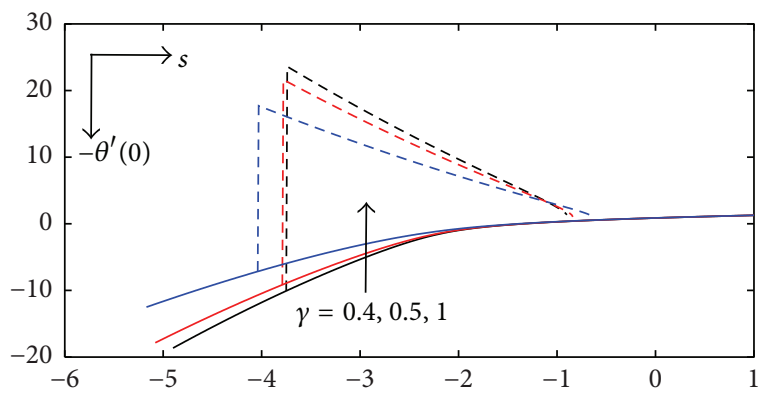

- Upper branch

- - - Lower branch

FIGURE 5: Variation of heat transfer at the surface with $s$ for various values of $\gamma$ with $M=0.5, \lambda=0.5$, and $\operatorname{Pr}=1$.

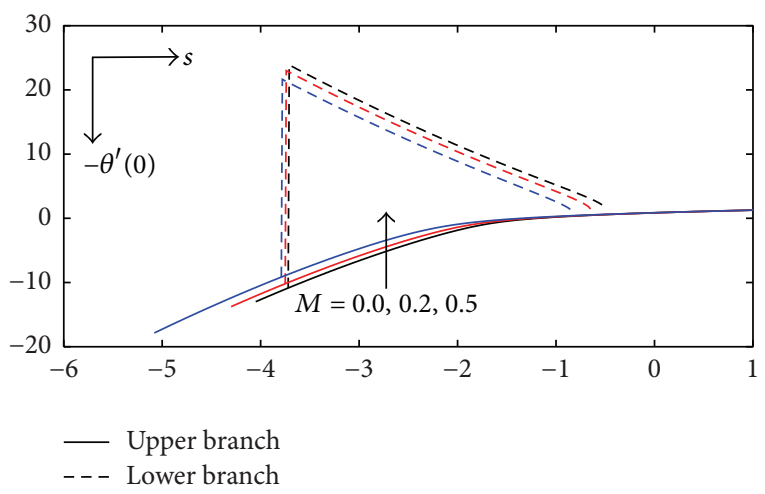

FIGURE 6: Variation of heat transfer at the surface with $s$ for various values of $M$ with $\gamma=0.5, \lambda=0.5$, and $\operatorname{Pr}=1$.

$-\theta^{\prime}(0)$ also decay with the increasing $M$ but profiles of $f^{\prime \prime}(0)$ observe opposite trends. Further, it is noted that the second solution is lesser numerically than the first solution for the case of $f^{\prime \prime}(0)$ whereas reverse patterns have been exhibited by the first and second solution profiles for the rate of heat transfer case. The main interesting result which we obtain from these figures is that the unique solutions are found to exist on both sides of dual solution range $\left(s_{1}, s_{2}\right)$ and hence extending the solution range tremendously.

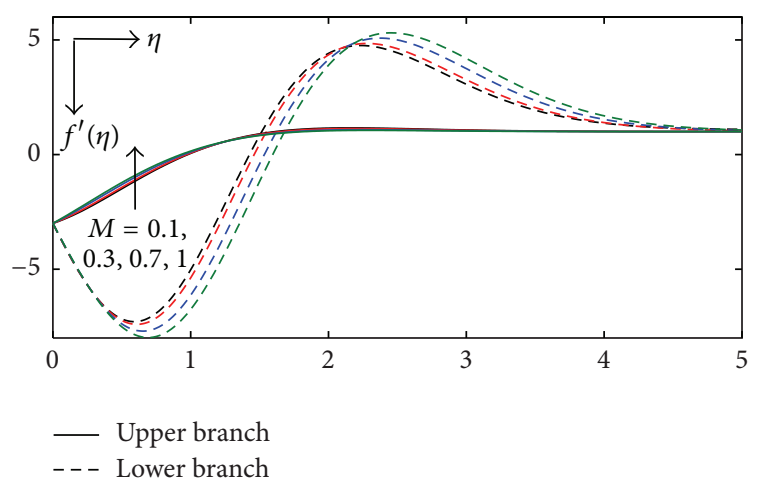

FIGURE 7: Velocity profiles for various values of $M$ with $s=-3, \gamma=$ $0.5, \lambda=1$, and $\operatorname{Pr}=3$.

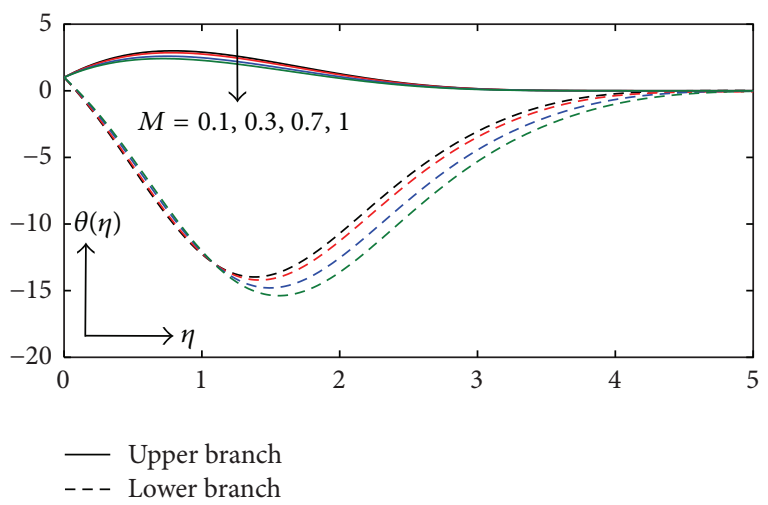

FIgURE 8: Temperature profiles for various values of $M$ with $s=-3$, $\gamma=0.5, \lambda=1$, and $\operatorname{Pr}=3$.

Figures 7 and 8 have been plotted, respectively, to discuss the influence of magnetic field $(M)$ on velocity $f^{\prime}(\eta)$ and temperature $\theta(\eta)$ profiles of the problem under consideration. Figure 7 depicts an increase in velocity profiles of upper and lower branch solutions with the increasing strength of the magnetic field, and the velocity boundary layer thickness is found to be decaying with the increasing $M$. This figure also reveals that increasing $M$ has more significant effect on the profiles of lower branch solutions as compared to the upper branch solutions. Further, at certain critical distance from the sheet some cross flow points have been observed for $f^{\prime}(\eta)$ and after these interception points reverse behavior is noted. The physics behind this phenomenon is that the magnetic field interaction can stabilize or destabilize the flow and for the present case a destabilizing influence has been noticed. Here, it is interesting to point out that lower branch profiles cross the upper branch ones; and all $f^{\prime}(\eta)$ curves are initially decreasing and attaining larger negative values and then start to increase for huge values of $\eta$, thereby attaining the positive values. This phenomenon shows reverse flow patterns which is physically due to the shrinking and straining velocities in opposite directions, a responsible factor for reverse flow. The effect of magnetic field $(M)$ is to decrease the upper branch temperature profiles without any cross flow points as Figure 8 exhibits, whereas lower branch solution profiles of $\theta(\eta)$ are 


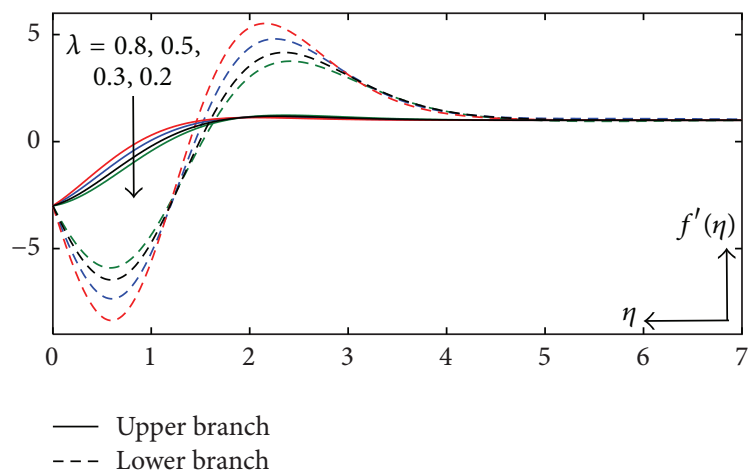

FIGURE 9: Velocity profiles for various values of $\lambda$ with $s=-3, \gamma=1$, $M=0.2$, and $\operatorname{Pr}=3$.

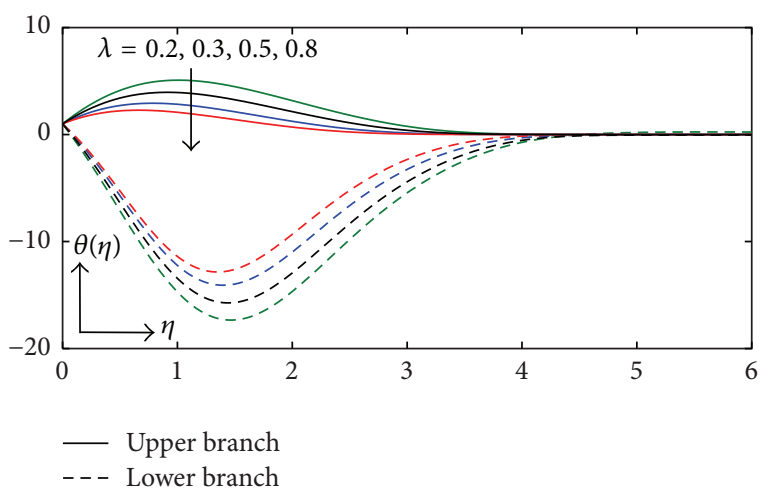

FIGURE 10: Temperature profiles for various values of $\lambda$ with $s=-3$, $\gamma=1, M=0.2$, and $\operatorname{Pr}=3$.

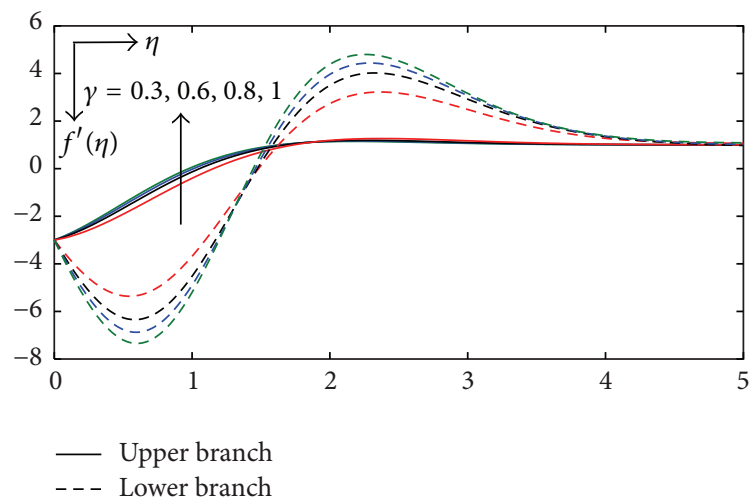

FIGURE 11: Velocity profiles for various values of $\gamma$ with $s=-3, \lambda=$ $0.5, M=0.2$, and $\operatorname{Pr}=3$.

initially increased and later on the pattern is reversed after crossing the critical point at a certain distance from the sheet. Here, the point of importance is that lower branch solutions are numerically lesser than upper branch solutions; and upper branch profiles approach the far field boundary conditions asymptotically from positive values whereas lower branch profiles approach them from negative values. Physically it means that magnetic field $(M)$ has a stabilizing effect on the temperature field $\theta(\eta)$.

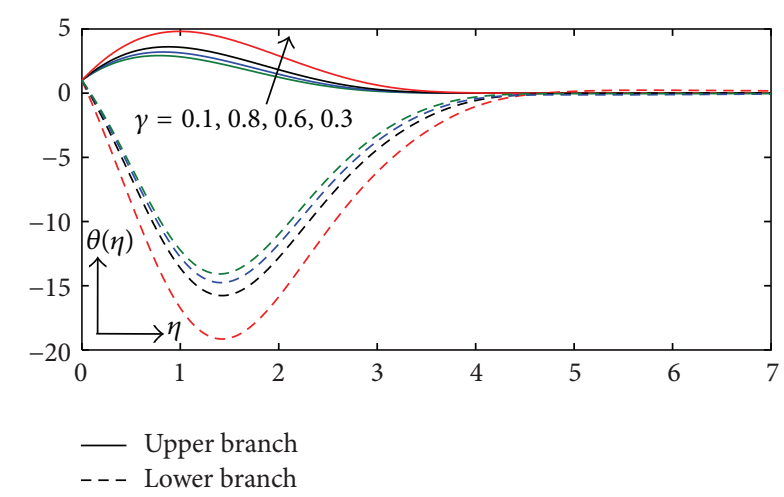

FIGURE 12: Temperature profiles for various values of $\gamma$ with $s=-3$, $\lambda=0.5, M=0.2$, and $\operatorname{Pr}=3$.

The distributions of velocity $f^{\prime}(\eta)$ and temperature $\theta(\eta)$ fields for different values of buoyancy parameter $(\lambda)$ have been illustrated, respectively, through Figures 9 and 10. For upper branch solutions, $f^{\prime}(\eta)$ increases and $\theta(\eta)$ decreases, whereas $f^{\prime}(\eta)$ are reduced and $\theta(\eta)$ are enhanced for lower branch solutions. Further, multiple interception points along with the reverse pattern after crossing them have been noticed for the upper and lower branch profiles of $f^{\prime}(\eta)$, and no cross flow points have been observed for that of $\theta(\eta)$. Again, the lower branch profiles cross the upper branch for $f^{\prime}(\eta)$ and the lower branch is always numerically lesser than the upper branch for $\theta(\eta)$. Moreover, in the vicinity of the sheet, the velocity gradients are negative for both of the branches whereas temperature gradient is positive for the upper branch and negative for the lower branch. It is interesting to communicate here that $\lambda$ significantly alters $f^{\prime}(\eta)$ and $\theta(\eta)$ for both of the branches.

The effects of nonlinear convection parameter $(\gamma)$ on the profiles of velocity $f^{\prime}(\eta)$ and temperature $\theta(\eta)$ have been displayed, respectively, by Figures 11 and 12. The influence of $\gamma$ on the distributions of $f^{\prime}(\eta)$ and $\theta(\eta)$ is quite similar to that of buoyancy parameter $(\lambda)$ with a difference that only one interception point has been noticed for upper and lower branch profiles of $f^{\prime}(\eta)$. The other difference observed is that nonlinear convection parameter has more pronounced effect on $\theta(\eta)$, whereas no significant effect has been observed for $f^{\prime}(\eta)$.

The Prandtl number (Pr) effects on velocity $f^{\prime}(\eta)$ and temperature $\theta(\eta)$ fields are presented in Figures 13 and 14. From Figure 13, it is observed that upper branch velocity profiles are increased and the corresponding boundary layer thickness becomes thinner with the increasing $\mathrm{Pr}$; however $\mathrm{Pr}$ has the opposite effect on the second branch solutions; that is, it decreases in the vicinity of the sheet. One interception point has also been noticed for lower branch solutions of $f^{\prime}(\eta)$ and after this point the flow trends are reversed. The significant effect of $\operatorname{Pr}$ on upper and lower branch profiles of $f^{\prime}(\eta)$ is revealed by Figure 13. It is interesting to note from this figure that the negative velocity gradients are converted into positive gradients for Prandtl number greater than or equal to 3 ( $P r \geq 3$ app.) for the upper branch solutions of $f^{\prime}(\eta)$ thereby making the flow physically 


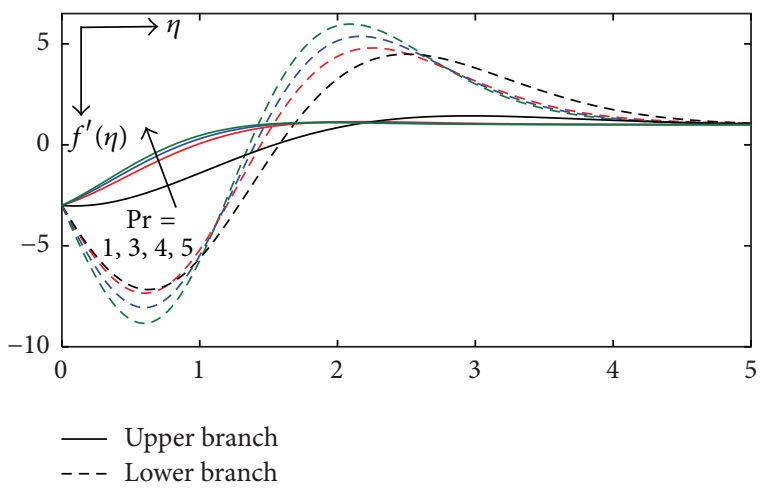

FIgURE 13: Velocity profiles for various values of $\operatorname{Pr}$ with $s=-3$, $\gamma=1, \lambda=0.5$, and $M=0.2$.

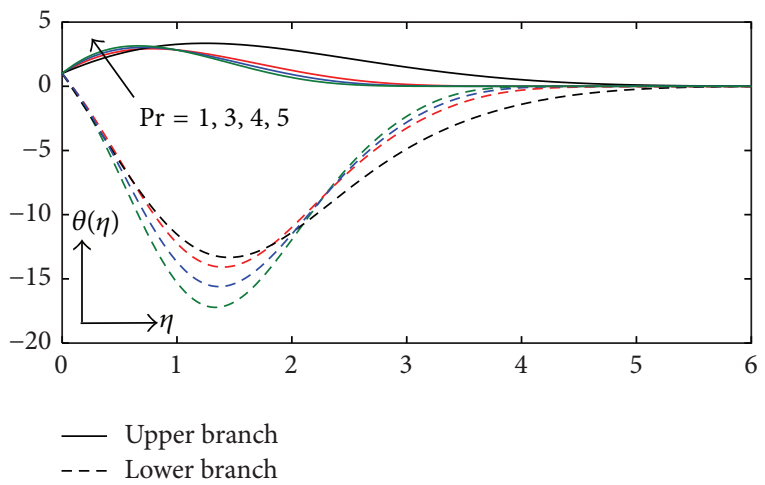

FIGURE 14: Temperature profiles for various values of $\operatorname{Pr}$ with $s=-3$, $\gamma=1, \lambda=0.5$, and $M=0.2$.

reliable. Also, since Prandtl number is the ratio of momentum diffusion to thermal diffusion, therefore, larger values of $\mathrm{Pr}$ represent the larger momentum diffusion which results in the enhancement of velocity profiles. On the other hand, from Figure 14 it is exhibited that the upper branch of $\theta(\eta)$ is increased and corresponding boundary layer is decayed with increasing $\operatorname{Pr}$ whereas the lower branch of $\theta(\eta)$ and boundary layer are reduced. This is due to the physical fact that larger Prandtl number represents a decreased thermal conductivity which results in a reduced thermal boundary layer thickness.

\section{Conclusions}

A study of the similarity solution of steady MHD stagnation point flow over a shrinking sheet with nonlinear convection was carried out. The main objective of this study was to analyze the influence of physical parameters involved in the system, namely, velocity ratio parameter, magnetic parameter, buoyancy parameter, NDT, and Prandtl number, on the solution. The implicit finite difference method has been employed to solve numerically the transformed nonlinear ordinary differential equations. Here, in this study dual solutions are found to exist. A new characteristic of our result came into sight, that is, presence of the severe gradients, and these severe gradients occur due to the presence of nonlinear convection. Additionally, it is observed that, with the increase in the magnetic field and the NDT, the dual solution region also increases.

\section{Competing Interests}

The authors declare that they have no competing interests.

\section{References}

[1] K. Bhattacharyya, T. Hayat, and A. Alsaedi, "Analytic solution for magnetohydrodynamic boundary layer flow of Casson fluid over a stretching/shrinking sheet with wall mass transfer," Chinese Physics B, vol. 22, no. 2, Article ID 024702, 2013.

[2] A. Chakrabarti and A. S. Gupta, "Hydromagnetic flow and heat transfer over a stretching sheet," Quarterly Journal of Applied Mathematics, vol. 37, pp. 73-78, 1979.

[3] Z. Zhang and J. Wang, "On the similarity solutions of magnetohydrodynamic flows of power-law fluids over a stretching sheet," Journal of Mathematical Analysis and Applications, vol. 330, no. 1, pp. 207-220, 2007.

[4] T. Kakutani, "Axially symmetric stagnation-point flow of an electrically conducting fluid under transverse magnetic field," Journal of the Physical Society of Japan, vol. 15, pp. 688-695, 1960.

[5] H. A. Attia, "Axisymmetric stagnation point flow towards a stretching surface in the presence of a uniform magnetic field with heat generation," Tamkang Journal of Science and Engineering, vol. 10, no. 1, pp. 11-16, 2007.

[6] F. M. Ali, R. Nazar, N. M. Arifin, and I. Pop, "MHD stagnationpoint flow and heat transfer towards stretching sheet with induced magnetic field," Applied Mathematics and Mechanics, vol. 32, no. 4, pp. 409-418, 2011.

[7] F. M. Ali, R. Nazar, N. M. Arifin, and I. Pop, "Mixed convection stagnation-point flow on vertical stretching sheet with external magnetic field," Applied Mathematics and Mechanics, vol. 35, no. 2, pp. 155-166, 2014.

[8] T. R. Mahapatra, S. K. Nandy, and A. S. Gupta, "Magnetohydrodynamic stagnation-point flow of a power-law fluid towards a stretching surface," International Journal of Non-Linear Mechanics, vol. 44, no. 2, pp. 123-128, 2009.

[9] U. Khan, N. Ahmed, S. I. Khan, and S. T. Mohyud-din, "Thermo-diffusion effects on MHD stagnation point flow towards a stretching sheet in a nanofluid," Propulsion and Power Research, vol. 3, no. 3, pp. 151-158, 2014.

[10] T. Hayat, Z. Abbas, and M. Sajid, "MHD stagnation-point flow of an upper-convected Maxwell fluid over a stretching surface," Chaos, Solitons and Fractals, vol. 39, no. 2, pp. 840-848, 2009.

[11] T. Hayat, A. Rafique, M. Y. Malik, and S. Obaidat, "Stagnationpoint flow of Maxwell fluid with magnetic field and radiation effects," Heat Transfer-Asian Research, vol. 41, no. 1, pp. 23-32, 2012.

[12] S. Shateyi and O. D. Makinde, "Hydromagnetic stagnationpoint flow towards a radially stretching convectively heated disk," Mathematical Problems in Engineering, vol. 2013, Article ID 616947, 8 pages, 2013.

[13] W. Ibrahim, B. Shankar, and M. M. Nandeppanavar, "MHD stagnation point flow and heat transfer due to nanofluid towards a stretching sheet," International Journal of Heat and Mass Transfer, vol. 56, no. 1-2, pp. 1-9, 2013.

[14] T. R. Mahapatra and A. S. Gupta, "Magnetohydrodynamic stagnation-point flow towards a stretching sheet," Acta Mechanica, vol. 152, no. 1-4, pp. 191-196, 2001. 
[15] A. Ishak, R. Nazar, N. M. Arifin, F. M. Ali, and I. Pop, "MHD stagnation-point flow towards a stretching sheet with prescribed surface heat flux," Sains Malaysiana, vol. 40, no. 10, pp. 1193-1199, 2011.

[16] Y. Y. Lok, N. Amin, and I. Pop, "Non-orthogonal stagnation point flow towards a stretching sheet," International Journal of Non-Linear Mechanics, vol. 41, no. 4, pp. 622-627, 2006.

[17] C. Y. Wang, "Stagnation flow towards a shrinking sheet," International Journal of Non-Linear Mechanics, vol. 43, no. 5, pp. 377$382,2008$.

[18] T. R. Mahapatra and S. K. Nandy, "Stability of dual solutions in stagnation-point flow and heat transfer over a porous shrinking sheet with thermal radiation," Meccanica, vol. 48, no. 1, pp. 2332, 2013.

[19] A. M. Rohni, S. Ahmad, and I. Pop, "Flow and heat transfer at a stagnation-point over an exponentially shrinking vertical sheet with suction," International Journal of Thermal Sciences, vol. 75, pp. 164-170, 2014.

[20] O. D. Makinde, W. A. Khan, and Z. H. Khan, "Buoyancy effects on MHD stagnation point flow and heat transfer of a nanofluid past a convectively heated stretching/shrinking sheet," International Journal of Heat and Mass Transfer, vol. 62, no. 1, pp. 526-533, 2013.

[21] K. Zaimi, A. Ishak, and I. Pop, "Boundary layer flow and heat transfer over a nonlinearly permeable stretching/shrinking sheet in a nanofluid," Scientific Reports, vol. 4, article 4404, 2014.

[22] N. S. Akbar, S. Nadeem, R. U. Haq, and S. Ye, "MHD stagnation point flow of Carreau fluid toward a permeable shrinking sheet: dual solutions," Ain Shams Engineering Journal, vol. 5, no. 4, pp. 1233-1239, 2014.

[23] L. Y. Yian, A. Ishak, and I. Pop, "MHD stagnation point flow with suction towards a shrinking sheet," Sains Malaysiana, vol. 40, no. 10, pp. 1179-1186, 2011.

[24] N. Bachok, A. Ishak, R. Nazar, and N. Senu, "Stagnation-point flow over a permeable stretching/shrinking sheet in a copperwater nano fluid," Boundary Value Problems, vol. 2013, article 39, 2013.

[25] S. Nadeem, S. Abbasbandy, and M. Hussain, "Series solutions of boundary layer flow of a micropolar fluid near the stagnation point towards a shrinking sheet," Zeitschrift fur Naturforschung A, vol. 64, no. 9, pp. 575-582, 2009.

[26] K. Bhattacharyya, M. G. Arif, and W. Ali Pramanik, "MHD boundary layer stagnation-point flow and mass transfer over a permeable shrinking sheet with suction/blowing and chemical reaction," Acta Technica, vol. 57, no. 1, pp. 1-15, 2012.

[27] R. A. Van Gorder, K. Vajravelu, and I. Pop, "Hydromagnetic stagnation point flow of a viscous fluid over a stretching or shrinking sheet," Meccanica, vol. 47, no. 1, pp. 31-50, 2012.

[28] S. Mansur, A. Ishak, and I. Pop, “The magnetohydrodynamic stagnation point flow of a nanofluid over a stretching/shrinking sheet with suction," PLoS ONE, vol. 10, no. 3, Article ID e0117733, 2015.

[29] A. Ishak, Y. Y. Lok, and I. Pop, "Stagnation point flow over a shrinking sheet in micropolar fluid," Chemical Engineering Communications, vol. 197, no. 11, pp. 1417-1427, 2010.

[30] K. Vajravelu and K. S. Sastri, "Fully developed laminar free convection flow between two parallel vertical walls-I," International Journal of Heat and Mass Transfer, vol. 20, no. 6, pp. 655-660, 1977.

[31] R. Bhargava and R. S. Agarwal, "Fully developed free convection flow in a circular pipe," Indian Journal of Pure and Applied Mathematics, vol. 10, pp. 357-365, 1979.
[32] K. Vajravelu, J. R. Cannon, J. Leto et al., "Nonlinear convection at a porous flat plate with application to heat transfer from a dike," Journal of Mathematical Analysis and Applications, vol. 277, no. 2, pp. 609-623, 2003.

[33] T. Cebeci and P. Bradshaw, Physical and Computational Aspects of Convective Heat Transfer, Springer, Berlin, Germany, 1984.

[34] H. B. Keller and T. Cebeci, "Accurate numerical methods for boundary-layer flows. II. Two-dimensional turbulent flows," The American Institute of Aeronautics and Astronautics Journal, vol. 10, pp. 1193-1199, 1972.

[35] H. B. Keller, "A new difference scheme for parabolic problems," in Numerical Solution of Partial Differential Equations-II, B. Hubbard, Ed., pp. 327-350, Academic Press, New York, NY, USA, 1970.

[36] T. R. Mahapatra, S. K. Nandy, and I. Pop, "Dual solutions in magnetohydrodynamic stagnation-point flow and heat transfer over a shrinking surface with partial slip," Journal of Heat Transfer, vol. 136, no. 10, Article ID 104501, 2014.

[37] M. Miklavcic and C. Y. Wang, "Viscous flow due to a shrinking sheet," Quarterly of Applied Mathematics, vol. 64, no. 2, pp. 283290, 2006. 


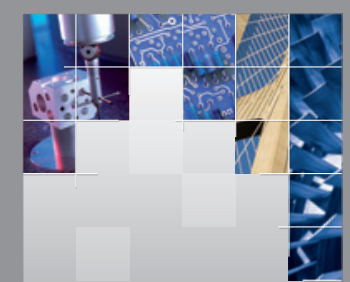

\section{Enfincering}
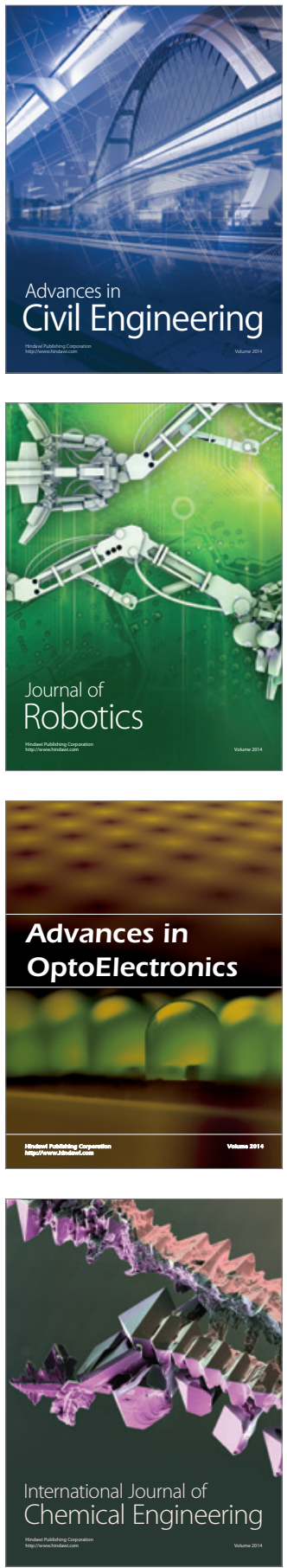

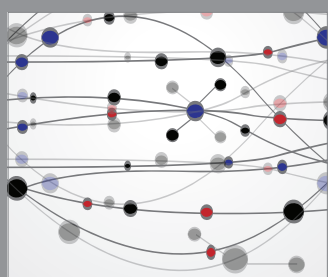

The Scientific World Journal

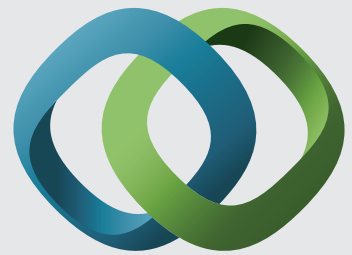

\section{Hindawi}

Submit your manuscripts at

http://www.hindawi.com
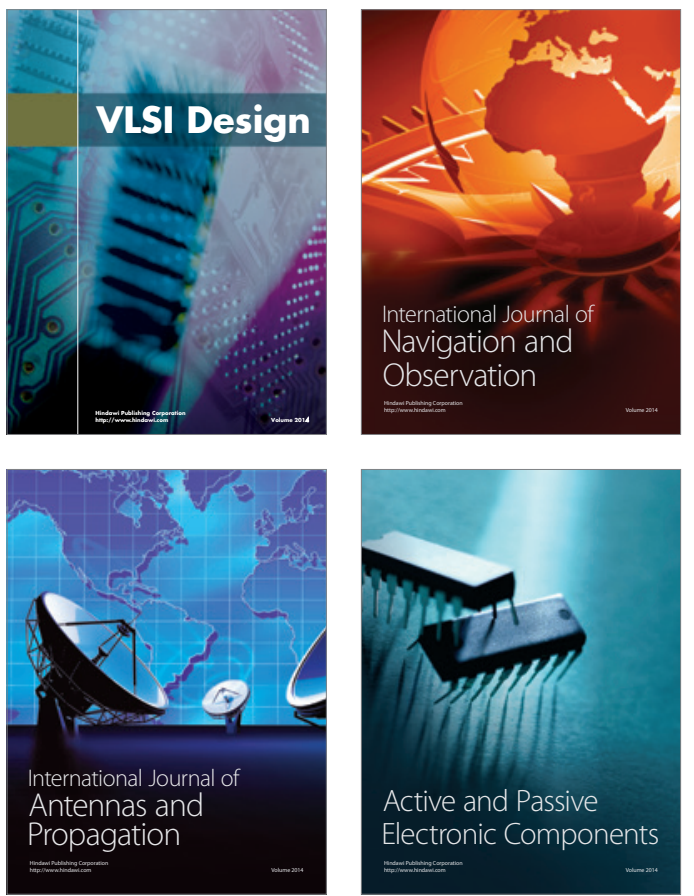
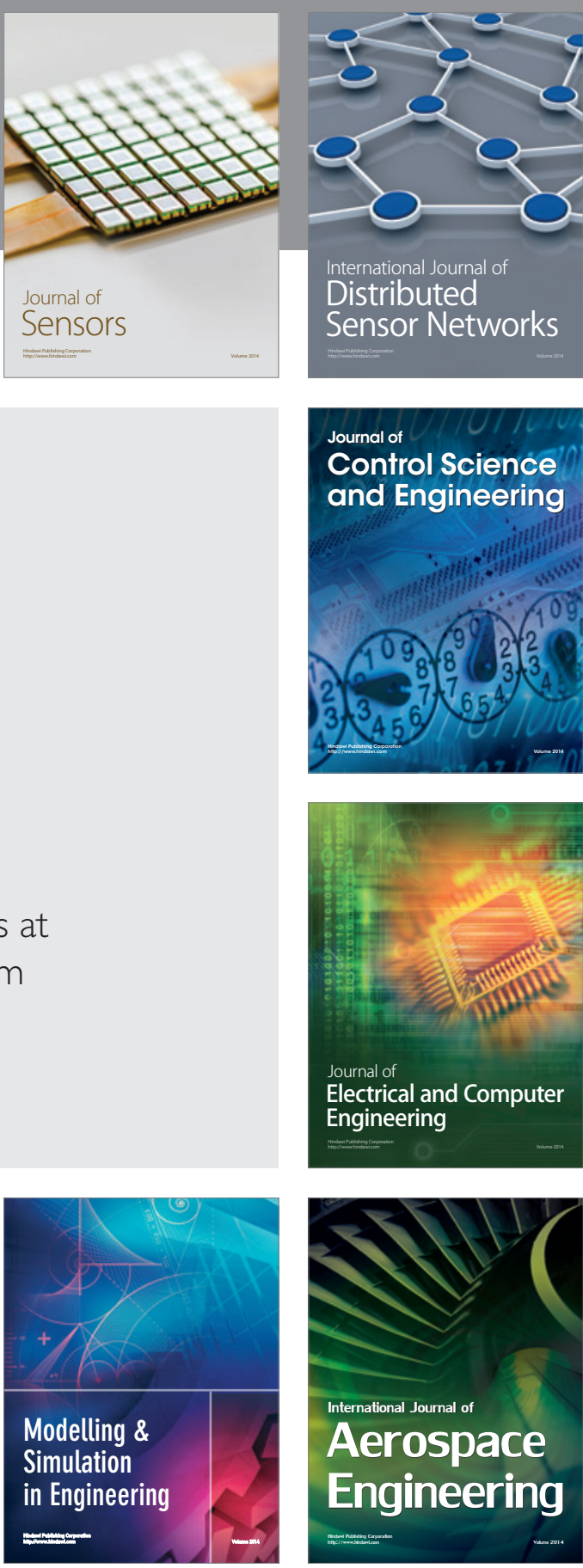

International Journal of

Distributed

Sensor Networks

Journal of

Control Science

and Engineering
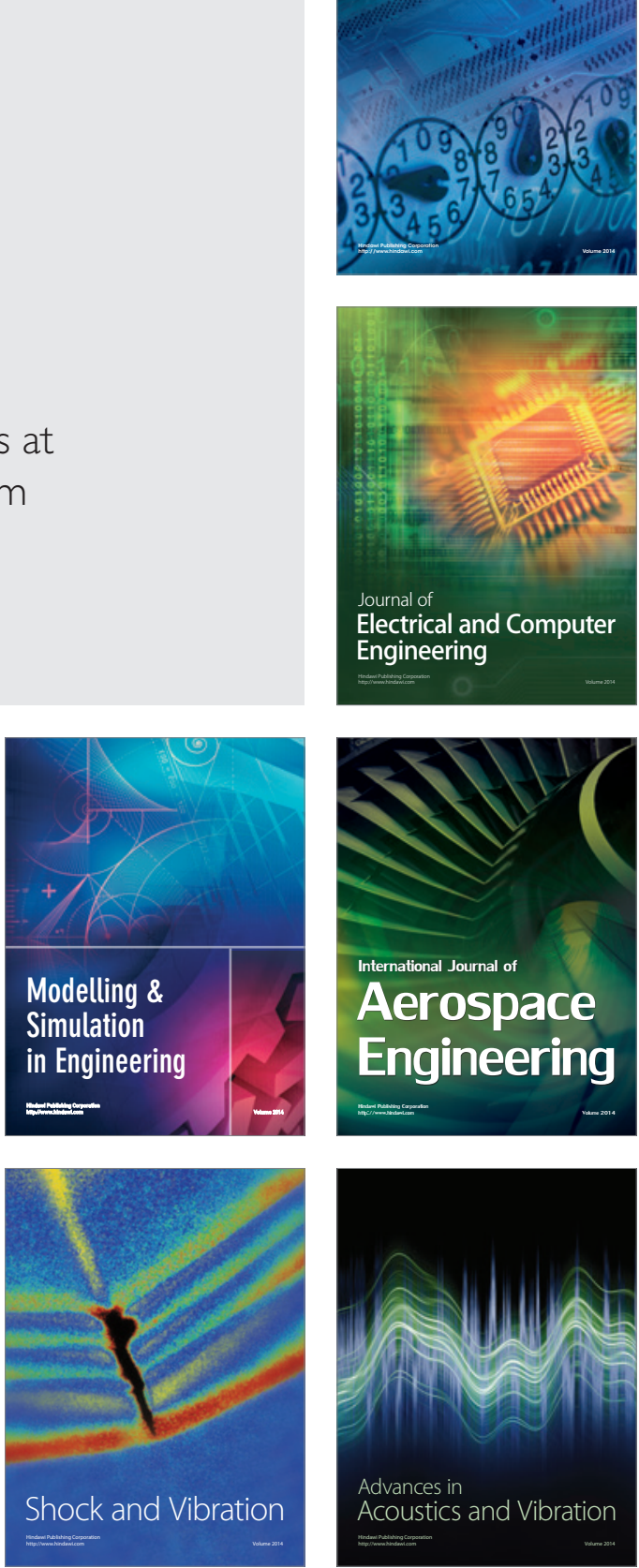